\title{
Traceable metallic antigen release for enhanced cancer immunotherapy
}

\author{
Fangfang Lv $\cdot$ Yan Jin $\cdot$ Xiaochen Feng $\cdot$ \\ Miao Fan $\cdot$ Cui Ren $\cdot$ Xinyue Dai $\cdot$ Jinchao Zhang $\cdot$ \\ Zhenhua Li $\cdot$ Yi Jin $\cdot$ Huifang Liu
}

Received: 10 December 2020 / Accepted: 1 June 2021 / Published online: 14 June 2021

(C) The Author(s), under exclusive licence to Springer Nature B.V. 2021

\begin{abstract}
Tumor vaccine has shown outstanding advantages and good therapeutic effects in tumor immunotherapy. However, antigens in tumor vaccines can be easily cleared by the reticuloendothelium system in advance, which leads to poor therapeutic effect of tumor vaccines. Moreover, it was still hard to monitor the fate and distribution of antigens. To address these limitations, we synthesized a traceable nanovaccine based on gold nanocluster-labeled antigens and upconversion nanoparticles (UCNPs) for the treatment of melanoma in this study. PH-sensitive Schiff base bond is introduced between UCNPs and gold
\end{abstract}

F. Lv $\cdot$ C. Ren $\cdot$ H. Liu $(\bowtie)$

College of Pharmaceutical Science, Key Laboratory of Pharmaceutical Quality Control of Hebei Province, Key Laboratory of Medicinal Chemistry and Molecular Diagnosis of Ministry of Education, Chemical Biology Key Laboratory of Hebei Province, Institute of Life Science and Green Development, Hebei University, Baoding 071002, China

e-mail: liu-huifang@163.com

Y. Jin $\cdot$ X. Feng $\cdot$ M. Fan $\cdot$ X. Dai $\cdot$ J. Zhang $\cdot$ Z. Li College of Chemistry \& Environmental Science, Institute of Life Science and Green Development, Key Laboratory of Medicinal Chemistry and Molecular Diagnosis of Ministry of Education, Chemical Biology Key Laboratory of Hebei Province, Hebei University, Baoding 071002, China

Y. Jin $(\bowtie)$

College of Basic Medical Science, Hebei University, Baoding 071000, China

e-mail: jinyi2009@163.com nanocluster-labeled ovalbumin antigens for monitoring antigens release. Our studies demonstrated that UCNPs conjugated metallic antigen showed excellent biocompatibility, $\mathrm{pH}$-sensitive and therapeutic effect.

Keywords Metallic antigen - Cancer vaccine · Immunotherapy $\cdot \mathrm{pH}$-sensitive $\cdot$ Gold nanoparticles

\section{Introduction}

Immunotherapy has become a promising method for treating many kinds of cancers, and many evidences indicated that immune system regulation was essential for tumor inhibition (Mahoney et al. 2015; Mellman et al. 2011; Vanneman and Dranoff 2012; Mi et al. 2019). The means of cancer immunotherapy include immune checkpoint inhibitors (Fan et al. 2018; Wieder et al. 2018), engineered chimeric antigen receptor $T$ cells (CAR-T) (Eisenberg et al. 2019), and tumor vaccines (Vormehr et al. 2019). Immune checkpoint inhibitors have achieved encouraging progress in clinical trials. However, it has been reported that only a small number of patients are benefitted from immune checkpoint inhibitors therapy (Kurtulus et al. 2019). CAR-T cell therapy also has serious clinical adverse reactions, such as cytokine storms (Zhang et al. 2017; Chen et al. 2019), targeted cytotoxicity, and a small portion of neurologic toxicity. The tumor vaccine has shown outstanding advantages and excellent therapeutic effects in immunotherapy with 
minimal systemic toxicity. Tumor vaccine regulates the immune function by activating the specific cellular immunity and humoral immunity and has been considered to be one of the most effective cancer treatments (Yang et al. 2019; Srinivasan et al. 2017). However, there are some problems with the cancer vaccine such as short blood circulation time and the antigen easy degradation.

In recent years, nanomaterials have been widely used as delivering carrier of tumor vaccines to improve the internalization by antigen-presenting cells (APC) (Han et al. 2018; Yoshikawa et al. 2008; Wang et al. 2018). It can be said that synthetic materials had the greatest impact on immunology through their contribution to vaccine development (Irvine 2016). Tumor-associated antigens (TAAs) can be either loaded inside the nanomaterial or conjugated to the surface of the nanomaterial through chemical modification. These works are designed to address the problem that antigens are easily degraded, while few of these focus on tracing the fate and biodistribution of vaccines.

The physical and chemical properties of the material affect the effect of vaccine in various ways (Briquez et al. 2020; DeSimone 2016). Therefore, we have to select suitable materials according to the needs of vaccine carriers and the physical and chemical properties of the materials. The application of rare-earth upconversion nanoparticles (UCNPs) has brought transformative opportunities and developments in the fields of diagnosis, treatment, imaging, and biological monitoring in the biomedical field (Wang et al. 2017; Liu et al. 2014; Zhou et al. 2020). Upconversion of rare-earth ions is an anti-stokes process of combining two or more low energy photons to produce the emission of one higher energy photon (Gao et al. 2017). Based on the special function of UCNPs, the introduction of UCNPs in tumor vaccines can not only improve the stability of blood circulation and prevent premature clearance by the reticuloendothelial system, but also track the location of antigens.

Here, we have prepared a traceable nanovaccine based on UCNPs and ovalbumin (OVA) for the treatment of melanoma (Scheme 1). Firstly, alendronate sodium (ALN) modified UCNPs and gold nanocluster labeled ovalbumin (Au-OVA) were synthesized, respectively, and then 1, 4-phthalaldehyde (TPDA) were used as bridging agents to integrate ALN-UCNPs and Au-OVA into a multifunctional nanosystem. UCNPs can convert NIR light into ultraviolet (UV) emissions which subsequently actives gold nanocluster to emit red fluorescence. More importantly, the Schiff base formed from ALN on UCNPs and $\mathrm{NH}_{2}$ of antigens is sensitive to acid, which ensures a $\mathrm{pH}$ - triggered antigen release. The disassembly of Au-OVA with UCNPs breaks FRET and turn off the red fluorescence. It ensures us to monitor the release of OVA from UCNP-Au-OVA system. We believe that our $\mathrm{pH}$-sensitive tumor tracing and antigen release system will open a new window for enhanced cancer immunotherapy.

\section{Materials and methods}

Materials

1-Octadecene and oleic acid were purchased from Thermo Fisher Scientific. $\mathrm{YCl}_{3} \cdot 6 \mathrm{H}_{2} \mathrm{O}, \mathrm{HAuCl}_{4}$, OVA, and trypsin were obtained from Sigma (St. Louis, MO, USA). $\mathrm{NH}_{4} \mathrm{~F}, \mathrm{ALN}$, and TPDZ were purchased from Aladdin (Shanghai, China). Fetal bovine serum was provided by Invitrogen (Carlsbad, CA, USA). Sodium bicarbonate, dibasic sodium phosphate, potassium phosphate monobasic, and potassium chloride were purchased from Kemiou (Tianjin, China). Calf serum was obtained from Wisent Canada. Penicillin-streptomycin solution 100X was obtained from Solarbio (Beijing, China). Four to five weeks C57BL/6 J mice (no. 1100111911071516) were obtained from Vital River (Beijing, China).

\section{Synthesis of UCNPs}

UCNPs were synthesized according to the literature with some modifications (Huang et al. 2012; Luo and Akimoto 2013; Tian et al. 2014). The $4 \mathrm{~mL}$ oleic acid, $6 \mathrm{~mL}$ 1-octadecene, $1 \mathrm{~mL} \mathrm{YCl}(0.2 \mathrm{M}), 0.98 \mathrm{~mL}$ $\mathrm{YbCl}_{3}(0.2 \mathrm{M})$, and $0.02 \mathrm{~mL} \mathrm{TmCl}(0.2 \mathrm{M})$ were added into a three-neck $50-\mathrm{mL}$ round bottom flask. The mixture was heated to $160{ }^{\circ} \mathrm{C}$ slowly, and then the solution was incubated with a certain rate stirring for $40 \mathrm{~min}$. After that the mixture was cooled down to room temperature. Next, the mixture was added with $\mathrm{NH}_{4} \mathrm{~F} \mathrm{NaOH}$ methanol solution $(\mathrm{NaOH} 1 \mathrm{~mL} 1 \mathrm{M}$, $\mathrm{NH}_{4} \mathrm{~F} 3.3 \mathrm{~mL} 0.4 \mathrm{M}$ ) dropwise and stirred for $30 \mathrm{~min}$ at $55{ }^{\circ} \mathrm{C}$. The mixture was degassed at $90{ }^{\circ} \mathrm{C}$ to remove methanol and then heated to $280{ }^{\circ} \mathrm{C}$ for $1.5 \mathrm{~h}$. 


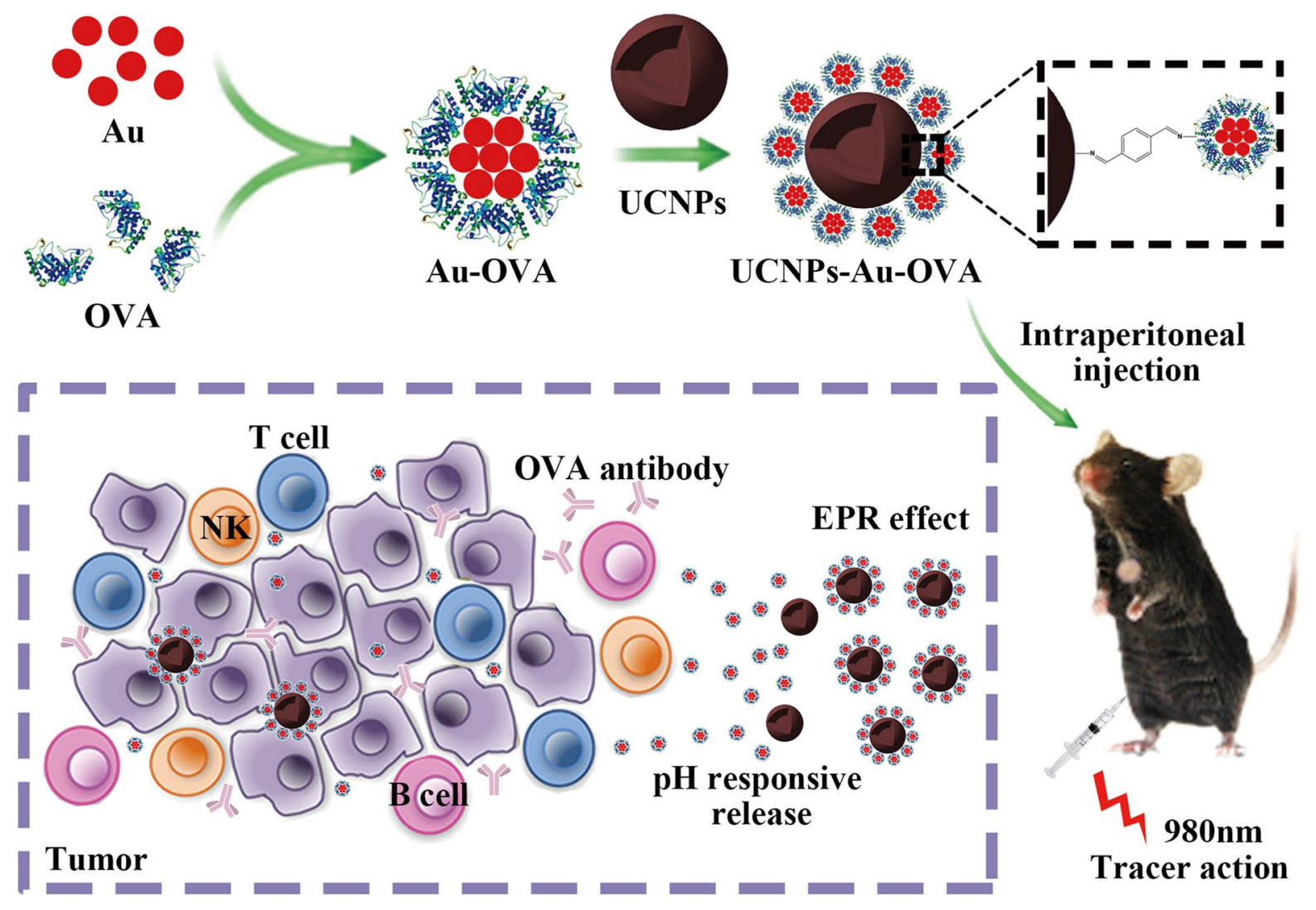

Scheme 1 A traceable nanovaccine based on UCNPs and ovalbumin (OVA) for the treatment of melanoma

When cooled down to room temperature, $\mathrm{NaYF}_{4}$ : Tm was obtained from the solution followed by washing with ethanol and cyclohexane several times. The obtained sample was dispersed in cyclohexane.

Synthesis of Au-OVA

$\mathrm{HAuCl}_{4}$ solution $(5 \mathrm{~mL}, 10 \mathrm{mM})$ was added to the vigorously stirred OVA solution $(5 \mathrm{~mL}, 50 \mathrm{mg} / \mathrm{mL})$. After a few minutes, $\mathrm{NaOH}$ solution was added into the mixture $(0.5 \mathrm{~mL}, 1 \mathrm{M})$ and stirred vigorously for $12 \mathrm{~h}$ at $37{ }^{\circ} \mathrm{C}$. A mixed solution of Au-OVA was obtained.

Synthesis of UCNPs-Au-OVA

A $50 \mathrm{mg}$ UCNPs was dispersed in $20 \mathrm{~mL}$ of chloroform and mixed with $40 \mathrm{~mL}$ of aqueous solution of $200 \mathrm{mg}$ ALN. The mixture was sonicated for $2 \mathrm{~h}$ and then stirred vigorously for $12 \mathrm{~h}$. The upper aqueous solution was sonicated vigorously and centrifuged and then washed 3 times with ultrapure water. After that, it was vacuum-dried to obtain UCNPs-ALN.

UCNPs-ALN was dispersed in excess of $0.1 \mathrm{M}$ solution of TPDA in ethanol, stirred for $1 \mathrm{~h}$ at room temperature, and then washed 3 times with ethanol and dried under vacuum to obtain UCNPs-ALNTPDA. An appropriate amount of UCNPs-ALNTPDA was added to the excess Au-OVA, stirred for $1 \mathrm{~h}$, washed with ultrapure water 3 times, and freezedried to obtain UCNPs-Au-OVA.

\section{Characterizations}

The morphology of UCNPs and Au-OVA was inspected by transmission electron microscope (TEM) (FEI F20 S-Twin, USA). The crystal purity of UCNPs was characterized by X-ray diffractometer (XRD) (Bruker D8 Advanced, Germany). The fluorescent 
properties of UCNPs and Au-OVA were characterized by fluorescence spectrometer (Hitachi F-7000, Japan). The absorption spectra of UCNPs, UCNPsALN, and UCNPs-ALN-TPDA were inspected by Fourier transform infrared (FTIR) spectrometers (Bruker TENSOR 27, Germany).

Validation of UCNPs-Au-OVA tracer function evaluation in vitro

RAW264.7 cells were cultured in a confocal petri dish at a density of $1 \times 10^{5}$ cells/well overnight. After that, UCNPs-ALN-TPDA (200 $\mu \mathrm{g} / \mathrm{mL})$ was added. The fluorescence intensity of cells was observed by confocal laser scanning microscopy at different time points (4 h, 24 h) (CLSM, IX81; Olympus, Tokyo, Japan).

Determination of loading efficiency (LE) and in vitro acid response release

The UCNPs-ALN-TPDA reacted with excess AuOVA, and the unloaded Au-OVA content in the supernatant was determined. Then, the LE of AuOVA is calculated according to Eq. 1 as follows: was centrifuged, and the absorbance at $546 \mathrm{~nm}$ was measured. The hemolysis rate is calculated according to the absorbance value.

Animal treatments and anti-tumor effect

The animal treatments were strictly proceeded in accordance with the regulation of the Medical Comprehensive Experimental Centre of Hebei University. B16-OVA tumor model was established with C57BL/6 J mice. B16-OVA cells were digested with trypsin and washed twice with normal saline, and the cell density was adjusted to $5 \times 10^{6}$ cells $/ \mathrm{mL}$. A $100 \mu \mathrm{L}$ B16-OVA cell suspension was subcutaneously inoculated on the right back of each mouse. The tumor-bearing mice were randomly divided into four groups (PBS, OVA, Au-OVA, and UCNPs-AuOVA). When the tumor size was approximately to 80 $\mathrm{mm}^{3}$, the mice were injected three times by intraperitoneal injection with $0.2 \mathrm{~mL}$ PBS, OVA $(1 \mathrm{mg} / \mathrm{mL})$, Au-OVA $(5 \mathrm{mg} / \mathrm{mL})$, and UCNPs-Au-OVA $(5 \mathrm{mg} /$ $\mathrm{mL}$ ) on days 0,7 , and 14 respectively. During the administration, we paid close attention to the physical health of the mice in all groups. The body weights

$\mathrm{LE}(\%)=[\mathrm{m}(\mathrm{Au}-$ OVATotal $)-\mathrm{m}(\mathrm{Au}-$ OVA supernatant $)] /[\mathrm{m}(\mathrm{Au}-$ OVA loading $)+\mathrm{m}(\mathrm{UCNPs})] \times 100 \%$

Take the same amount of UCNPs-Au-OVA, and add them to the buffer solutions of $\mathrm{pH}=5$ and $\mathrm{pH}=7$ respectively. Their fluorescence intensity at $0 \mathrm{~h}, 0.5 \mathrm{~h}$, $1 \mathrm{~h}, 2 \mathrm{~h}, 4 \mathrm{~h}$, and $8 \mathrm{~h}$ were measured by fluorescence spectrometer three times.

\section{Blood compatibility}

A $2 \mathrm{~mL}$ of fresh blood from ICR mice was collected in anticoagulant-pretreated blood collection tubes. The collected red blood cells were washed enough times with normal saline until the supernatant was clear and transparent. Finally, $0.2 \mathrm{~mL} 10 \%$ red blood cell suspension was mixed with $0.8 \mathrm{~mL}$ of UCNPsAu-OVA solution with different concentrations (12.5, $25,50,100,200 \mu \mathrm{g} / \mathrm{mL}$ ) as experimental groups. The positive control group was red blood cell suspension mixed with water $(0.2 \mathrm{~mL}$ with $0.8 \mathrm{~mL})$, and the negative control group was red blood cell suspension mixed with normal saline $(0.2 \mathrm{~mL}$ with $0.8 \mathrm{~mL})$. After incubation for $20 \mathrm{~min}$ at $37^{\circ} \mathrm{C}$, the supernatant and tumor volumes of the mice were measured every 2 days, and the tumor volume is calculated according to Eq. 2 as follows:

$\mathrm{V}=\mathrm{D}_{\max } * \mathrm{D}_{\min }^{2} / 2$.

$\mathrm{D}_{\text {max }}$ and $\mathrm{D}_{\text {min }}$ represent the maximum and minimum diameters, respectively.

\section{Results and discussion}

Characterization of UCNPs-Au-OVA

The morphology of UCNPs and Au-OVA were characterized by TEM. As shown in Fig. 1a, UCNPs were relatively uniform with a size of about $30 \mathrm{~nm}$. As shown in Fig. 1b, the diameter of Au-OVA particles was about $5 \sim 8 \mathrm{~nm}$ by statistical analysis. The XRD pattern of UCNPs (Fig. 1c) was consistent with the standard card (JCPD: 16-0334). All the results 

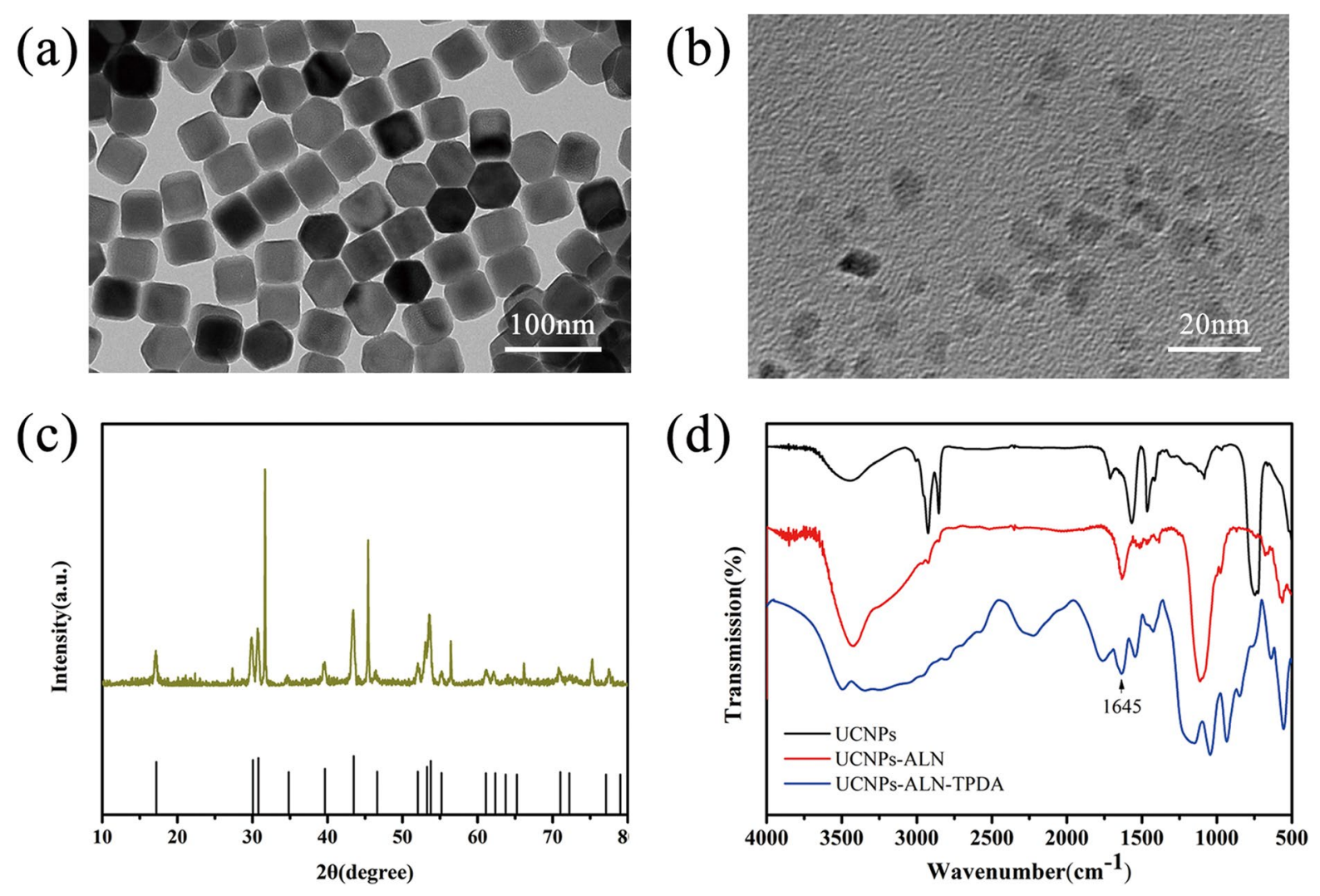

Fig. 1 Characterizations of UCNPs and Au-OVA. a TEM image of UCNPs. b TEM image of Au-OVA. c XRD pattern of UCNPs. d FTIR spectra of UCNPs, UCNPs-ALN, and UCNPs-ALN-TPDA

showed that UCNPs had high crystal purity and uniform size. Then, UCNPs, UCNPs-ALN, and UCNPsALN-TPDA were characterized by FTIR respectively. As shown in Fig. 1d, the absorption peaks at $1645 \mathrm{~cm}^{-1}$ corresponding with the $\mathrm{C}=\mathrm{N}$ group proved the successful modification of ALN with TPDA. Then, the LE of Au-OVA was measured, and the result showed that the LE was $26.29 \%$.

$\mathrm{Next}$, the fluorescence properties of UCNPs, $\mathrm{Au}-$ OVA, and UCNPs-Au-OVA were measured. Stokes' law states that materials can only be excited by highenergy light to emit low-energy light. But upconversion luminescence is anti-Stokes luminescence. When UCNPs is excited by low-energy light, it can emit high-energy light. As shown in Fig. 2a, the fluorescence can be detected around $360 \mathrm{~nm}$ and $480 \mathrm{~nm}$ when UCNPs were excited at $980 \mathrm{~nm}$. The UV absorption spectrum of Au-OVA showed that fluorescence generated at $360 \mathrm{~nm}$ could excite Au-OVA (Fig. 2d). The fluorescence spectrum of Au-OVA showed that the fluorescence emission wavelength of Au-OVA is $735 \mathrm{~nm}$ (red light) (Fig. 2b). As shown in Fig. 2c, when UCNPs-Au-OVA were excited at $980 \mathrm{~nm}$ in acidic environment, fluorescence can be detected only around $480 \mathrm{~nm}$, indicating that UCNPsAu-OVA have acid-responsive properties.

To further verify the acid-responsive properties of UCNPs-Au-OVA, we examined the fluorescence intensity of UCNPs-Au-OVA under neutral ( $\mathrm{pH}$ 7.0) and acid ( $\mathrm{pH}$ 5.0) environments. As shown in Fig. 2e, the fluorescence intensity under neutral environments was higher than that in acid environments. The results showed that part of Au-OVA could be dissociated in acidic environment. Therefore, these results further proved that UCNPs-Au-OVA had $\mathrm{pH}$ sensitivity characteristics. Due to the abnormal blood vessel structure and the excessive proliferation of tumor cells, tumor tissue is usually in a state of hypoxia, resulting in changes in its metabolic process. Therefore, solid tumors usually exhibit an acidic microenvironment 

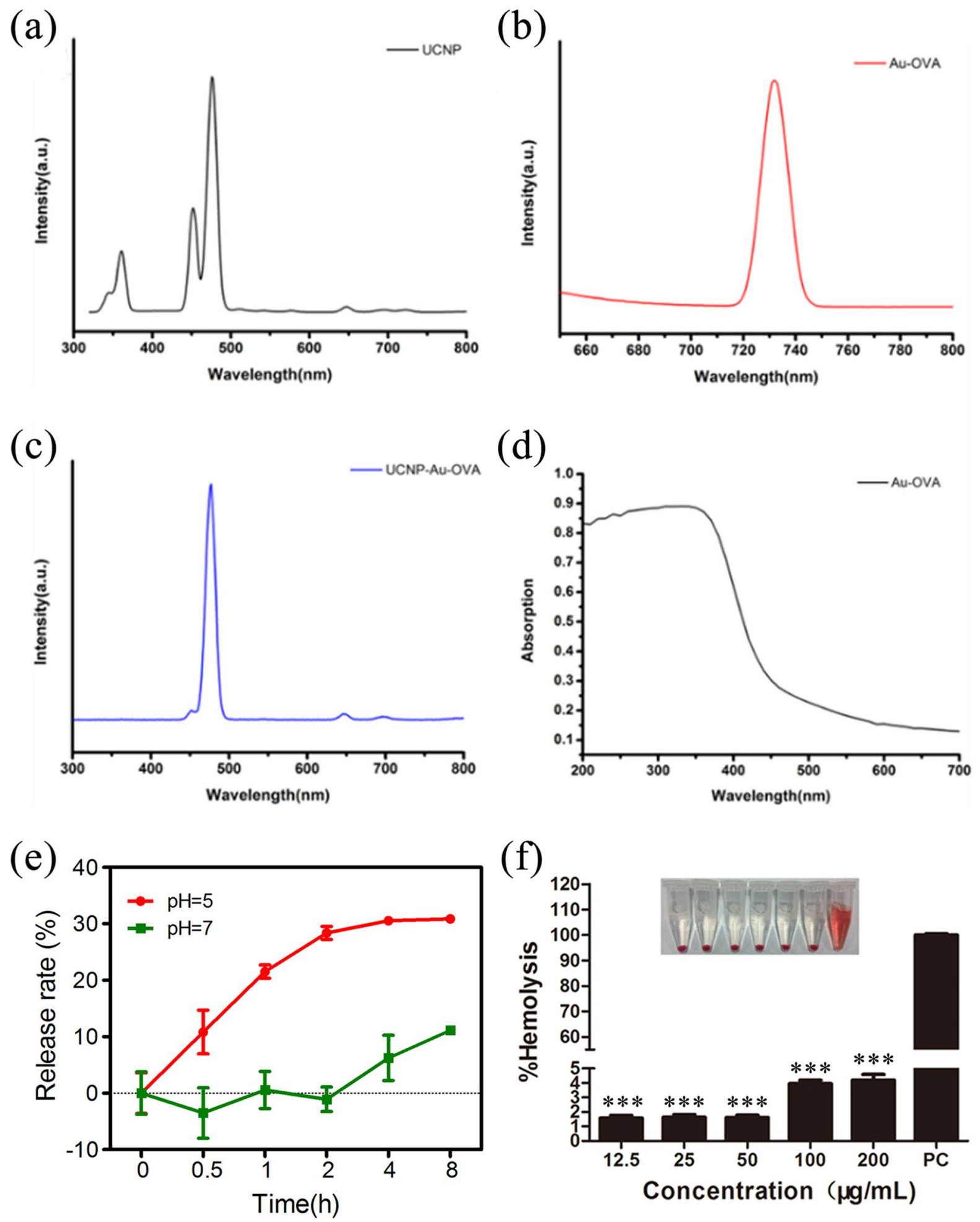

(f)

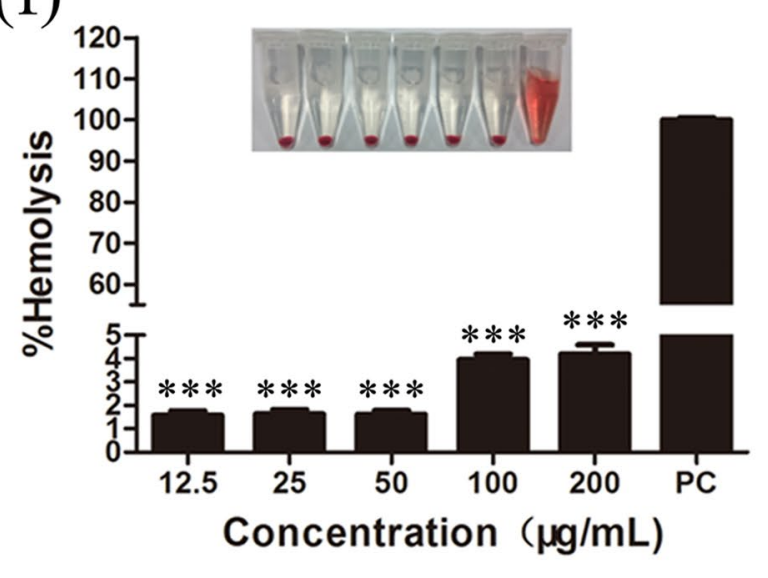

Fig. 2 pH-responsive property and biocompatibility of UCNPs-Au-OVA nanoparticles. a Fluorescence spectra of UCNPs. b Fluorescence spectra of Au-OVA. c Fluorescence spectra of UCNPs-Au-OVA under acidic conditions. d UV

absorption spectra of Au-OVA. e The release curve of UCNPsAu-OVA at different $\mathrm{pH}$. f Hemolysis assay of UCNPs-AuOVA $(* * * \mathrm{p}<0.001$ versus the positive control) 
(Böhme and Bosserhoff 2016; Feng et al. 2018; Kraus and Wolf 1996). A part of the imine bonds broke in the slightly acidic tumor microenvironment, and most of them were taken up by macrophages and then broke in the lysosome to release OVA, thereby activating the body's immune system to eliminate tumor cells.

\section{Evaluation of tracer function of UCNPs-Au-OVA}

We verified the tracer effect of UCNPs-Au-OVA at the cell level using CLSM. As shown in Fig. 3a, the macrophages without UCNPs-Au-OVA did not have any red fluorescence. The red fluorescence was observed in macrophages after $4 \mathrm{~h}$ incubation with UCNPs-Au-OVA. However, the red fluorescence was very weak after $24 \mathrm{~h}$ (Fig. 3b-c). It showed that UCNPs-Au-OVA had a definite tracing effect within $24 \mathrm{~h}$. This tracer function could be used to monitor the release of OVA, so as to better perform the immunotherapy function.
Upconversion luminescence, namely, anti-Stokes luminescence, means that the material is excited by low energy light and emits high energy light. In other words, the material emits short wavelength and high frequency light excited by long wavelength and low frequency light. UCNPs are the functional materials that can convert low-energy photons into high-energy photons. In view of the ability of UCNPs to convert NIR light into UV light or visible light, it has become one of the research hotspots in the field of biomedicine (Oliveira et al. 2019; Sharipov et al. 2017). The traceability of UCNPs is quite considerable for the research of cancer therapy. For example, Liu et al. used UCNPs as tracer to monitor the translocation of dendritic cells (DC) which ingested DC vaccines and revealed the anti-tumor process of DC vaccines (Xiang et al. 2015). Zhao et al. had developed PLGA nanocapsules based on UCNPs, which can be used for cancer imaging and treatment (Zhao et al. 2017). $\mathrm{NaYF}_{4}: \mathrm{Tm}$, as a tracer agent, can be excited with $980 \mathrm{~nm}$ lasers and shows upconverted fluorescence at

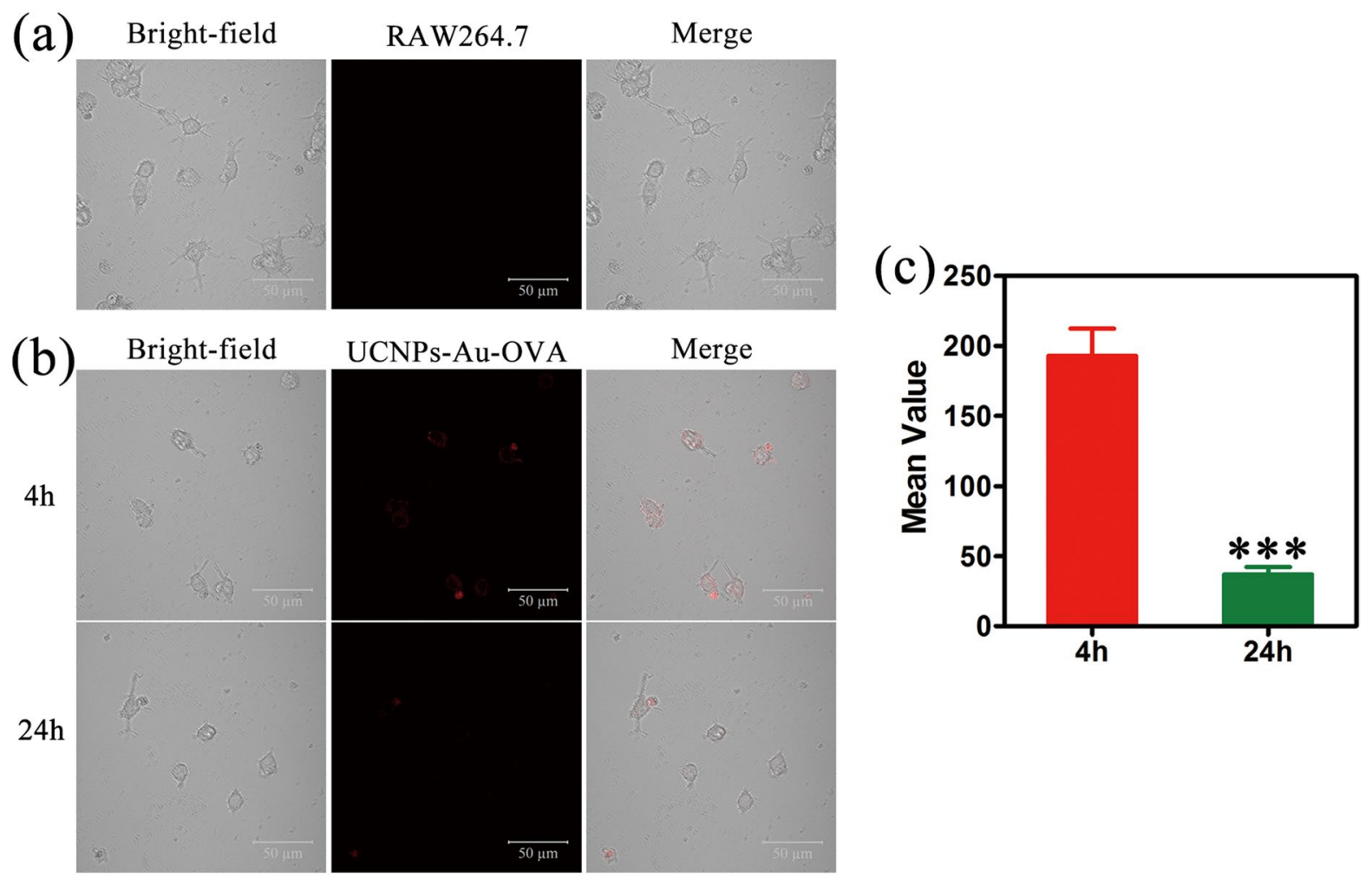

Fig. 3 Traceability of UCNPs-Au-OVA. a The fluorescence of macrophages without UCNPs-Au-OVA. b The fluorescence of macrophages after incubation with UCNPs-Au-OVA. c Fluorescence intensity of macrophages $(* \mathrm{p}<0.05, * * \mathrm{p}<0.01, * * * \mathrm{p}<0.001)$ 
$480 \mathrm{~nm}$ and $360 \mathrm{~nm}$, which can be attributed to the ${ }^{1} \mathrm{G}_{4} \rightarrow{ }^{3} \mathrm{H}_{6}$ and ${ }^{1} \mathrm{D}_{2} \rightarrow{ }^{3} \mathrm{H}_{6}$ transition of the $\mathrm{Tm}^{3+}$ ions, respectively. The excitation of $980 \mathrm{~nm}$ has excellent biological tissue penetration ability (Sharipov et al. 2017). However, the emission of $480 \mathrm{~nm}$ and $360 \mathrm{~nm}$ has poor penetrating ability in biological tissues. Therefore, the imaging of deep tissue is not possible. Therefore, we used $360 \mathrm{~nm}$ that UCNPs generated to excite Au-OVA, and the emission of Au-OVA was at $735 \mathrm{~nm}$. So the penetrating ability of UCNPs-AuOVA in biological tissues was improved and could be used to imaging of deep tissue.

\section{Biocompatibility evaluation of UCNPs-Au-OVA}

When exogenous nanomaterials are used in living organisms, rejection reactions can cause side effects. Truong et al. analyzed the interaction of nanoparticles with cells during blood circulation, indicating the importance of nanomaterial biocompatibility ( $\mathrm{Vu}$ et al. 2020). Therefore, the biocompatibility of UCNPs-Au-OVA was characterized by hemolysis assay. We tested the blood compatibility of UCNPsAu-OVA at different concentrations. It could be seen from Fig. $2 \mathrm{f}$ that the UCNPs-Au-OVA had good blood compatibility especially when the concentration less than $100 \mu \mathrm{g} / \mathrm{mL}$. Therefore, UCNPs-AuOVA had better biocompatibility and lower toxicity.

Anti-tumor effect of UCNPs-Au-OVA in vivo

As shown in Fig. 4a-c, the tumors of control group grew rapidly. The tumor volume of the OVA group was smaller compared with the control group. It indicated that OVA itself has weak anti-tumor effect. Moreover, tumor growth of the Au-OVA group was slower than that of control and OVA groups. Among all groups, the tumor size of the UCNPsAu-OVA group grew the slowest. The results indicated that UCNPs-Au-OVA had excellent tumor immunotherapy.

OVA, as a common model tumor antigen, was often used in the construction of tumor vaccines. However, tumor vaccines had the problems of short blood circulation time and easy degradation of antigens. Using nanomaterials as a carrier could solve the above problems (Mi et al. 2019). Loading TAAs with nanomaterials could not only promote the uptake of antigen by APC, but also protect the antigen from the surrounding biological environment. Moreover, it prolonged the blood circulation time of the antigen (Cheng et al. 2015). In addition, through the EPR effect, more antigens were enriched in the tumor tissue (Goos et al. 2020). Thereby, using nanomaterials as a carrier ensured that the adaptive immune response was fully triggered and the role of immunotherapy was exerted.

Evaluation of immune response

UCNPS-Au-OVA as a tumor vaccine could activate the immune system. OVA as vaccine antigens could activate B cells to produce specific antibodies (Mohankrishnan et al. 2019). We evaluated the total antigen-specific IgG, IgG2a, and IgG1 produced from four different groups (Fig. 4d). Au-OVA and UCNPs-Au-OVA induced a high IgG response and a high ratio of $\mathrm{IgG} 2 \mathrm{a} / \mathrm{IgG} 1$. It indicated that the tumor vaccine not only induced the production of specific antibodies, but also activated Th1-type immune responses.

NK cells and $\mathrm{CD}^{+} \mathrm{T}$ cells played a significant role in tumor immunotherapy (Wu and $\mathrm{Xu}$ 2010; Rosenberg and Huang 2018; Zhou et al. 2018). CD8 ${ }^{+}$ $\mathrm{T}$ cells killed cancer cells by releasing cytokines such as granzyme, perforin, and tumor necrosis factor. NK cells could also release killing cytokines and lyse some tumor cells, which was very central factor in tumor immunotherapy. Therefore, we analyzed the infiltration of $\mathrm{NK}$ cells and $\mathrm{CD}^{+} \mathrm{T}$ cells by analyzing tumor tissue sections. The increased NK cells and $\mathrm{CD}^{+} \mathrm{T}$ cells in tumor tissues could effectively inhibit tumor growth. At the end of the 21-day treatment period, the $\mathrm{CD} 8^{+} \mathrm{T}$ cell marker $\mathrm{CD} 8 \alpha$ and the NK cell marker CD161 were observed. As shown in Fig. $4 \mathrm{e}$ and $\mathrm{f}$, the number of NK cells and $\mathrm{CD} 8^{+}$ $\mathrm{T}$ cells in mice tumors increased significantly after treatment with UCNPs-Au-OVA.

\section{Conclusions}

In summary, we have synthesized a tumor vaccine with $\mathrm{pH}$ response and tracer effect for tumor immunotherapy through a multi-step method. First, we synthesized UCNPs according to previous literature reports, and then we formed the final multifunctional nanomaterials UCNPs-Au-OVA by the sequential 
(a)

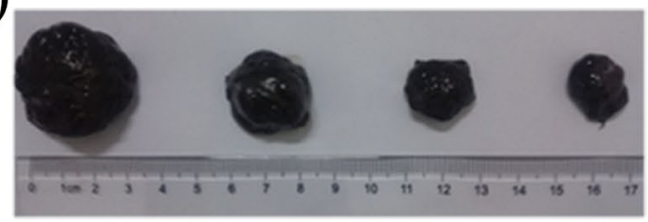

(b)

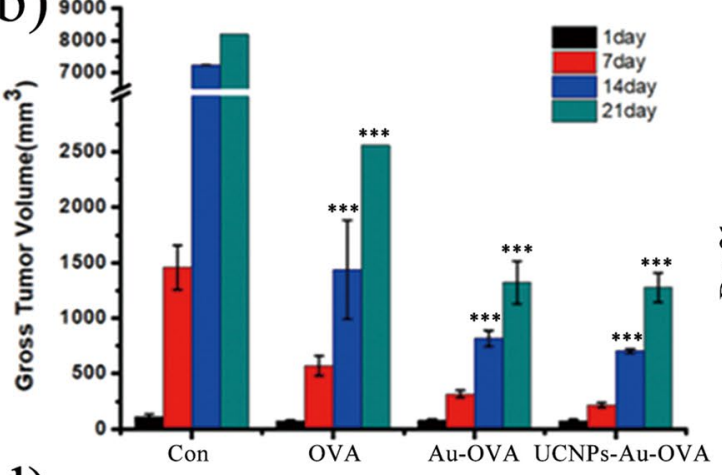

(d)

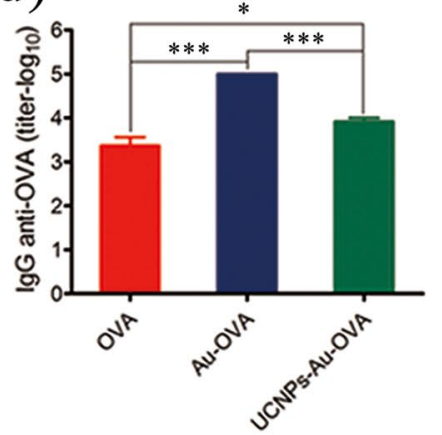

(f)

OVA (c)

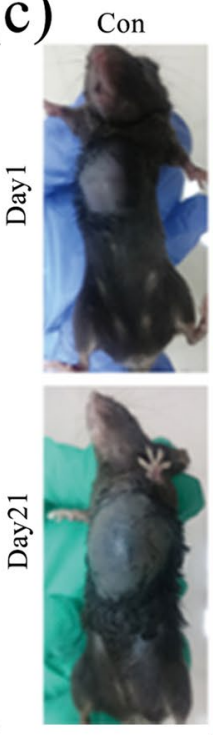

OVA

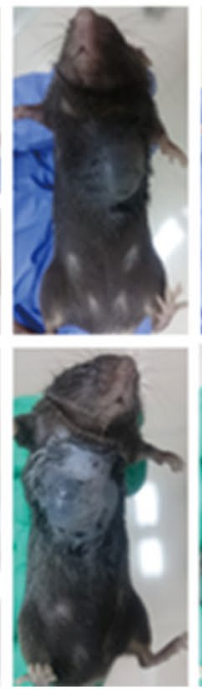

(e)

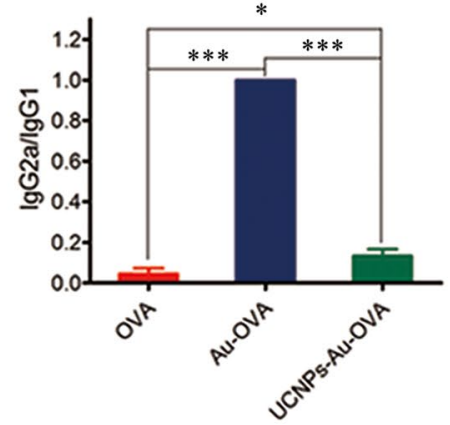

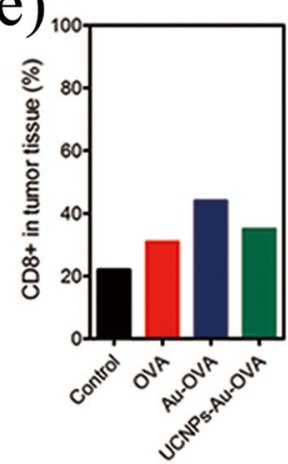

Au-OVA

UCNPs-Au-OVA
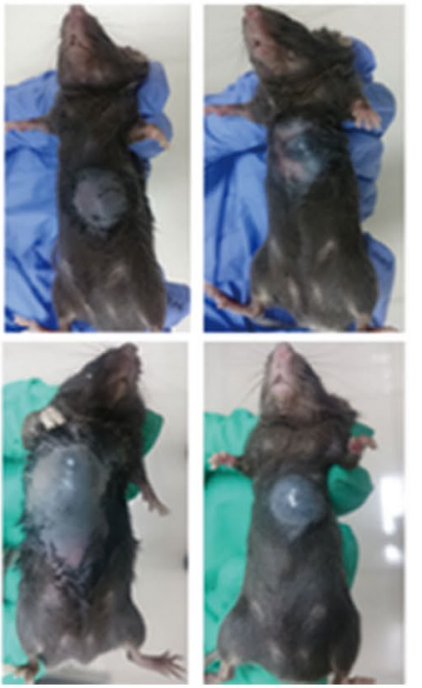



Au-OVA

UCNPs-Au-OVA

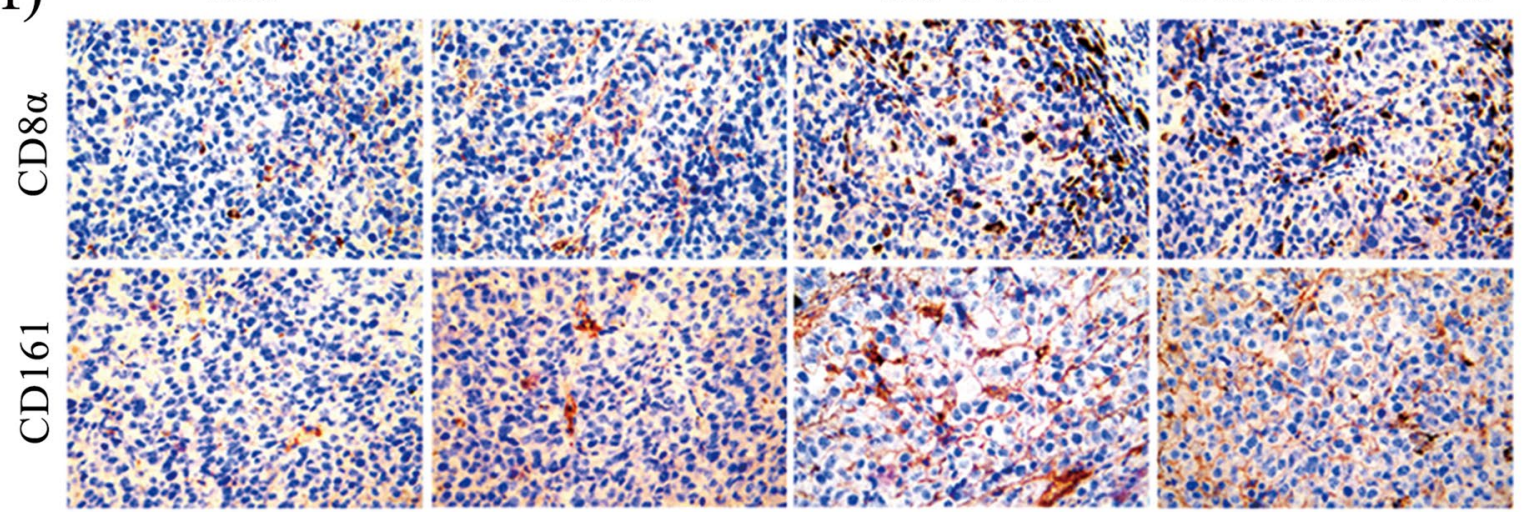

Fig. 4 Detection of anti-tumor effects of OVA, Au-OVA and UCNPs-Au-OVA in tumor-bearing mice. a Excised tumor image. b Tumor volume changes in each group $(* * * p<0.001$ versus the control). $\mathbf{c}$ Images of mice at the start and endpoint of the immunotherapy. d ELISA results of anti-OVA IgG and IgG $2 \mathrm{a} / \mathrm{IgG} 1$ titers elicited by different stimulus $(* \mathrm{p}<0.05$, $* * \mathrm{p}<0.01, * * * \mathrm{p}<0.001)$. e,f Immunohistochemical analysis of tumor explants for infiltrating $\mathrm{CD}^{+} \mathrm{T}$ cells and NK cells 
connection of ALN, TPDA, and Au-OVA. UCNPsAu-OVA could regulate the body's immune response and inhibit tumor growth through the acid-responsive release of Au-OVA. The results showed that UCNPs-Au-OVA had excellent biocompatibility and exceptional optical properties. In vivo experiments, UCNPs-Au-OVA showed a significant killing effect on tumor cells. Compared with other tumor vaccines, UCNPs-Au-OVA had the ability to track antigens, which can better achieve tumor immunotherapy.

Acknowledgements We are grateful to Medical Comprehensive Experimental Center of Hebei University for the animal experiment.

Funding This work was supported by the Natural Science Foundation of Hebei Province (B2018201185, B2018201157, B2020201091), Key Projects of Education Department of Hebei Province (ZD2018036), Advanced Talents Incubation Program of the Hebei University (801260201020), and Talent Training Program of Hebei University (521000981169, 521000981196).

\section{Declarations}

Conflict of interest The authors declare that they have no conflict of interests.

Animal and human rights consent The animal experiments were strictly proceeded in accordance with the regulation of the Medical Comprehensive Experimental Center of Hebei University (Approval No. 2018004).

\section{References}

Böhme I, Bosserhoff AK (2016) Acidic tumor microenvironment in human melanoma. Pigm Cell Melanoma R 29(5):508-523. https://doi.org/10.1111/pcmr.12495

Briquez PS, Hauert S, Titta A, Gray LT, Alpar AT, Swartz MA et al (2020) Engineering targeting materials for therapeutic cancer vaccines. Front Bioeng Biotechnol 8:19. https:// doi.org/10.3389/fbioe.2020.00019

Chen H, Wang FX, Zhang PY, Zhang YL, Chen YX, Fan XH et al (2019) Management of cytokine release syndrome related to CAR-T cell therapy. Front Med 13(5):610-617. https://doi.org/10.1007/s11684-019-0714-8

Cheng TJ, Ma RJ, Zhang YM, Ding YX, Liu JJ, Ou HL et al (2015) A surface-adaptive nanocarrier to prolong circulation time and enhance cellular uptake. Chem Commun 51(81):14985-14988. https://doi.org/10.1039/c5cc05854f

DeSimone JM (2016) Co-opting Moore's law: therapeutics, vaccines and interfacially active particles manufactured via PRINT. J Control Release. https://doi.org/10.1016/j. jconrel.2016.07.019
Eisenberg V, Hoogi S, Shamul A, Barliya T, Cohen CJ (2019) T-cells "à la CAR-T(e)" - Genetically engineering T-cell response against cancer. Adv Drug Deliv Rev 141:23-40. https://doi.org/10.1016/j.addr.2019.01.007

Fan Q, Chen ZP, Wang C, Liu Z (2018) Toward biomaterials for enhancing immune checkpoint blockade therapy. Adv Funct Mater 28(37):1802540-1802564. https://doi. org/10.1002/adfm.201802540

Feng LZ, Dong ZL, Tao DL, Zhang YC, Liu Z (2018) The acidic tumor microenvironment: a target for smart cancer nano-theranostics. Natl Sci Rev 5(2):269-286. https://doi.org/10.1093/nsr/nwx062

Gao GJ, Turshatov A, Howard IA, Busko D, Joseph R, Hudry $D$ et al (2017) Up-Conversion fluorescent labels for plastic recycling: a review. Adv Sustain Syst 1(5):16000331600058. https://doi.org/10.1002/adsu.201600033

Goos JACM, Davydova M, Dilling TR, Cho A, Cornejo MA, Gupta A et al (2020) Design and preclinical evaluation of nanostars for the passive pretargeting of tumor tissue. Nucl Med Biol 84-85:63-72. https://doi.org/10.1016/j. nucmedbio.2020.02.012

Han JY, Zhao DD, Li D, Wang XH, Jin Z, Zhao K (2018) Polymer-based nanomaterials and applications for vaccines and drugs. Polymers 10(1):31-44. https://doi.org/ 10.3390/polym 10010031

Huang WJ, Lu CH, Jiang CF, Wang W, Song JB, Ni TR et al (2012) Controlled synthesis of $\mathrm{NaYF}_{4}$ nanoparticles and upconversion properties of $\mathrm{NaYF}_{4}: \mathrm{Yb}, \mathrm{Er}(\mathrm{Tm}) / \mathrm{FC}$ transparent nanocomposite thin films. J Colloid Interf Sci 376(1):34-39. https://doi.org/10.1016/j.jcis.2012.02. 047

Irvine DJ (2016) Materializing the future of vaccines and immunotherapy. Nat Rev Mater 1(1):15008. https://doi. org/10.1038/natrevmats.2015.8

Kraus M, Wolf B (1996) Implications of Acidic Tumor microenvironment for neoplastic growth and cancer treatment: a computer analysis. Tumor Biol 17(3):133-154. https://doi. org/10.1159/000217977

Kurtulus S, Madi A, Escobar G, Klapholz M, Nyman J, Christian E et al (2019) Checkpoint blockade immunotherapy induces dynamic changes in $\mathrm{PD}-1^{-} \mathrm{CD} 8^{+}$tumor-infiltrating T cells. Immunity 50(1):181-194. https://doi.org/10. 1016/j.immuni.2018.11.014

Liu XD, Zhang X, Tian G, Yin WY, Yan L, Ruan LF et al (2014) A simple and efficient synthetic route for preparation of $\mathrm{NaYF}_{4}$ upconversion nanoparticles by thermodecomposition of rare-earth oleates. Cryst Eng Comm 16(25):5650-5661. https://doi.org/10.1039/c4ce00231h

Luo XJ, Akimoto K (2013) Upconversion properties in hexagonal-phase $\mathrm{NaYF}_{4}$ : $\mathrm{Er}^{3+} / \mathrm{NaYF}_{4}$ nanocrystals by off-resonant excitation. Appl Surf Sci 273(15):257-260. https:// doi.org/10.1016/j.apsusc.2013.02.026

Mahoney KM, Rennert PD, Freeman GJ (2015) Combination cancer immunotherapy and new immunomodulatory targets. Nat Rev Drug Discov 14(8):561-584. https://doi.org/ 10.1038/nrd4591

Mellman I, Coukos G, Dranoff G (2011) Cancer immunotherapy comes of age. Nature 480(7378):480-489. https://doi. org/10.1038/nature10673

Mi Y, Hagan IVCT, Vincent BG, Wang AZ (2019) Emerging nano-/microapproaches for cancer immunotherapy. Adv 
Sci 6(6):1801847-1801869. https://doi.org/10.1002/advs. 201801847

Mohankrishnan A, Patel H, Bhurani V, Parmar R, Yadav N, Dave $N$ et al (2019) Inclusion of Non-target antigen in vaccination favors generation of OVA specific CD4 Memory $\mathrm{T}$ cells. Cell Immunol 337:1-14. https://doi.org/10. 1016/j.cellimm.2018.11.005

Oliveira H, Bednarkiewicz A, Falk A, Fröhlich E, Lisjak D, Prina-Mello A et al (2019) Critical considerations on the clinical translation of upconversion nanoparticles (UCNPs): recommendations from the European upconversion network (COST Action CM1403). Adv Healthc Mater 8(1):1801233-1801242. https://doi.org/10.1002/ adhm.201801233

Rosenberg J, Huang J (2018) CD8 ${ }^{+}$T cells and NK cells: parallel and complementary soldiers of immunotherapy. Curr Opin Chem Eng 19:9-20. https://doi.org/10.1016/j.coche. 2017.11.006

Sharipov M, Tawfik SM, Gerelkhuu Z, Huy BT, Lee YI (2017) Phospholipase A2-responsive phosphate micelle-loaded UCNPs for bioimaging of prostate cancer cells. Sci Rep 7(1):16073-16081. https://doi.org/10.1038/s41598-017-16136-4

Srinivasan VM, Ferguson SD, Lee S, Weathers SP, Parker Kerrigan BC, Heimberger AB (2017) Tumor vaccines for malignant gliomas. Neurotherapeutics 14(2):345-357. https://doi.org/10.1007/s13311-017-0522-2

Tian XN, Jiang GC, Wei XT, Wu LY, Li S, Deng KM et al (2014) Synthesis and photoluminescent properties of $\mathrm{NaYF}_{4}: \mathrm{Eu}^{3+}$ core and $\mathrm{NaYF}_{4}: \mathrm{Eu}^{3+} / \mathrm{NaYF}_{4}$ core/shell nanocrystals. J Nanosci and Nanotechno 14(6):44904494. https://doi.org/10.1166/jnn.2014.8053

Vanneman M, Dranoff G (2012) Combining immunotherapy and targeted therapies in cancer treatment. Nat Rev Cancer 12(4):237-251. https://doi.org/10.1038/nrc3237

Vormehr M, Türeci Ö, Sahin U (2019) Harnessing tumor mutations for truly individualized cancer vaccines. Annu Rev Med 70(1):395-407. https://doi.org/10.1146/annur ev-med-042617-101816

Vu MN, Kelly HG, Wheatley AK, Peng S, Pilkington EH, Veldhuis NA et al (2020) Cellular interactions of liposomes and pisa nanoparticles during human blood flow in a microvascular network. Samll 2002861. https:// doi.org/10.1002/smll.202002861

Wang XD, Valiev RR, Ohulchanskyy TY, Ågren H, Yang CH, Chen GY (2017) Dye-sensitized lanthanide-doped upconversion nanoparticles. Chem Soc Rev 46(14):4150-4167. https://doi.org/10.1039/c7cs00053g
Wang JM, Hu XB, Xiang DX (2018) Nanoparticle drug delivery systems: an excellent carrier for tumor peptide vaccines. Drug Deliv 25(1):1319-1327. https://doi.org/10. 1080/10717544.2018.1477857

Wieder T, Eigentler T, Brenner E, Rocken M (2018) Immune checkpoint blockade therapy. J Allergy Clin Immun 142(5):1403-1414. https://doi.org/10.1016/j.jaci.2018.02. 042

Wu ZF, Xu YB (2010) IL-15R $\alpha$-IgG1-Fc Enhances IL-2 and IL-15 Anti-tumor action through $\mathrm{NK}$ and $\mathrm{CD} 8^{+} \mathrm{T}$ cells proliferation and activation. J Mol Cell Biol 2(4):217222. https://doi.org/10.1093/jmcb/mjq012

Xiang J, Xu LG, Gong H, Zhu WW, Wang C, Xu J et al (2015) Antigen-loaded upconversion nanoparticles for dendritic cell stimulation, tracking, and vaccination in dendritic cell-based immunotherapy. ACS Nano 9(6):6401-6411. https://doi.org/10.1021/acsnano.5b02014

Yang WJ, Zhu GZ, Wang S, Yu GC, Yang Z, Lin L et al (2019) In situ dendritic cell vaccine for effective cancer immunotherapy. ACS Nano 13(3):3083-3094. https://doi.org/10. 1021/acsnano.8b08346

Yoshikawa T, Okada N, Oda A, Matsuo K, Matsuo K, Kayamuro $\mathrm{H}$ et al (2008) Nanoparticles built by self-assembly of amphiphilic $\gamma$-PGA can deliver antigens to antigen-presenting cells with high efficiency: A new tumor-vaccine carrier for eliciting effector T cells. Vaccine 26(10):13031313. https://doi.org/10.1016/j.vaccine.2007.12.037

Zhang C, Liu J, Zhong JF, Zhang X (2017) Engineering CAR-T cells. Biomark Res 5(1):22-27. https://doi.org/10.1186/ s40364-017-0102-y

Zhao JW, Yang H, Li JL, Wang YJ, Wang X (2017) Fabrication of pH-responsive PLGA(UCNPs/DOX) nanocapsules with upconversion luminescence for drug delivery. Sci Rep 7(1):18014 18024. https://doi.org/10.1038/s41598-017-16948-4

Zhou XM, Li WQ, Wu YH, Han L, Cao XG, Yang XM et al (2018) Intrinsic expression of immune checkpoint molecule TIGIT could help tumor growth in vivo by suppressing the function of $\mathrm{NK}$ and $\mathrm{CD} 8^{+} \mathrm{T}$ cells. Front Immunol 9:2821-2831. https://doi.org/10.3389/fimmu.2018.02821

Zhou B, Li QQ, Yan L, Zhang QY (2020) Controlling upconversion through interfacial energy transfer (IET): fundamentals and applications. J Rare Earths 38(5):474-482. https://doi.org/10.1016/j.jre.2020.01.009

Publisher's Note Springer Nature remains neutral with regard to jurisdictional claims in published maps and institutional affiliations. 